Barcelona, Spain, November 17-20, 2013

\title{
Application of the Thermal Impedance Spectroscopy method in three dimensions to a large prismatic Li-ion cell
}

\author{
Pierre-Luc Lapointe ${ }^{1}$, \\ ${ }^{1}$ Institut du Transport Avancé du Québec,475 Fournier St-Jérôme, Québec, Canada, J7Z4V2,plapointe@ @stj.qc.ca
}

\begin{abstract}
Thermal impedance spectroscopy (TIS) is applied for the thermal characterization of a large prismatic battery cell. The method is based on previously published work and is extended to a three dimensional system. By using a discretized three dimensional model, the experimental results can be fitted to obtain the specific heat capacity and heat conductivity of the cell in three dimensions. The results show good agreement with the available data in the literature. Suggestions are made to improve the test setup to gain more confidence in the results and to increase the precision of the model.
\end{abstract}

Thermal impedance spectroscopy, Li-ion battery thermal modelling

\section{Introduction}

Many authors already proved that diminishing the thermal gradient within a Li-Ion battery assembly can improve the performance and the duration of the battery cells [1]. This concept is even more important in large assemblies such as those used in hybrid cranes and ferries where the power and capacity are larger than usual. The tall dimensions of such assemblies lead to increased magnitude of thermal gradients and can lead to increased cell aging or safety issues.

To face such challenges, designers and engineers relied a lot on thermal simulation of the battery cell. The modelling and simulation of the assembly can be based on Finite Difference, Finite Volume or Finite Element Method. The parameterization of such model can be a major source of inaccuracies. A proper method to characterise the thermal parameters of Li-ion battery cells is needed. The cost of such process should be kept as low as possible to test a wide selection of battery cell early in the design process.
The design process of a large Li-ion battery pack with one of the ITAQ (Institut du Transport Avancé du Québec) partner led to the necessity of developing the Thermal Impedance Spectroscopy method with a three dimensional model to find the thermal parameters of a high capacity prismatic cell.

Previous authors already proved that the method is working with pouch [2] and cylindrical cells [3] to find the thermal impedance spectrum or to use the impedance spectrum to fit thermal characteristics to a model. With this in mind, we tried to build upon the previous work of these groups to develop a 3 dimensional model that could be used to find the heat capacity and the heat conductivity for multiple directions. The results from this study will help the battery pack designers to improve the thermal management unit for their large pack in order to reduce the temperature gradient, decrease the balancing needs and reduce the differences in the life span of the cells. The chosen cell type was prismatic. 


\section{Theory}

Impedance spectroscopy is a common tool to study the behaviour in many fields of science [4]. It is referred as Electrochemical Impedance spectroscopy (EIS) when it is used to describe the transfer behaviour of electrochemical systems such as batteries and fuel cells. There is two methods available to obtain the impedance : the time-domain method and the frequency-domain method. The usual method, the frequencydomain method, stimulates the system at different frequencies to measure the response. By dividing the response $U(i \omega)$ by the stimulation signal $I(i \omega)$, we obtain the frequency-dependant impedance $Z(i \omega)$ :

$$
Z(i \omega)=\frac{U(i \omega)}{I(i \omega)}
$$

with $i \omega=i 2 \pi f$ where $i$ is the imaginary unit and $f$ is the frequency. $Z(i \omega), U(i \omega)$ and $I(i \omega)$ are complex numbers.

In the time-domain method, the time domain signal is processed through a Fourier transform to obtain frequency-domain data. The Fast Fourier transformation is usually applied since it is an efficient algorithm available in most numerical computing environments.

The first method is faster, since there is only one test needed to find the impedance while the second is easier since there is no heavy calculation involved.

For the thermal impedance spectroscopy, the input signal is chosen as the heat generation within the cell and the response is the cell surface temperature.

$$
G(i \omega)=\frac{T_{\text {surf }}(i \omega)}{Q(i \omega)}
$$

Since the time-domain method was chosen in the present test, we will derive the frequency dependant temperature $T_{\text {surf }}(i \omega)$ and heat generation $Q(i \omega)$ by means of the Fourier transform from the time domain signals $T_{\text {surf }}(t)$ and $Q(t)$ with the Fast Fourier transformation (FFT) with Matlab.
There are many methods to generate heat within the cell. The first one is to install a heating band wounded around the cell [5]. With this method, the thermocouple has to be placed in the centre of the cell. This is not representative of the conditions in which the cell will be used.

Another method is to use the power loss inside the cell to generate the heat as described in $[2,3]$. The heat flow is stimulated by a sinusoidal current or a square wave current. The chosen frequency should only stimulate processes given by ohmic loss contributions (electrolyte, electrodes and current collectors) to simplify the heat generation model. With only ohmic losses, the following equation is valid to describe the heat generation [6] :

$$
Q(t)=I(t)|(U-V(t))|
$$

Where $I(t)$ is the current, $U$ is the voltage under open circuit voltage condition and $V(t)$ is the voltage as a function of time.

The cell to be tested has a large capacity at $42 \mathrm{Ah}$ which implies that the current has to be large enough to generate heat. Since the researchers had access to material able to deliver such currents, the internal heat generation method was chosen.

\section{Model}

In order to obtain the thermal parameters $k_{x}, k_{y}, k_{z}$ and $c_{p}$, the measured data has to be fitted to a model. We chose to build and solve the model in a similar fashion as the nodal analysis used to solve electrical circuit problems. In this type of problem, the potential of each node (in our case, the temperature) is chosen as the unknown. The unknown potential is multiplied by an admittance matrix (the conductivity and heat capacity) and equals the generated current in each node, which in the present case is the heat generation. To build the admittance matrix, the Fourier law described in equation (4) was discretized with the Finite Volume method. [7]

$$
\begin{aligned}
c_{p} \cdot \frac{d T(t)}{d t}=k_{x} & \frac{d T(t)}{d x}+k_{y} \frac{d T(t)}{d y} \\
& +k_{z} \frac{d T(t)}{d z}+Q(t)
\end{aligned}
$$




$$
\begin{aligned}
& C_{p} \cdot \rho \cdot v_{x, y, z} T_{x, y, z}=\frac{Q}{n}+k_{x A}\left(T_{x+1, y, z}-T_{x, y, z}\right)+k_{y A}\left(T_{x, y+1, z}-T_{x, y, z}\right)+ \\
& k_{z A}\left(T_{x, y, z+1}-T_{x, y, z}\right)+\left(h_{A x}+h_{A y}+h_{A z}\right)\left(T_{x, y, z}-T_{\text {ambient }}\right)
\end{aligned}
$$

The heat capacity $\left(c_{p}\right)$, the heat conductivity $\left(k_{x}, k_{y}, k_{z}\right)$ and the density $(\rho)$ are considered homogeneous. The Laplace Transform is applied to the Fourier equation to transfer the model from time-domain to frequency domain :

$$
\begin{aligned}
& c_{p} \cdot T(s)-T(0) \\
& =k_{x} \frac{d T(s)}{d x}+k_{y} \frac{d T(s)}{d y}+k_{z} \frac{d T(s)}{d z} \\
& +Q(s)
\end{aligned}
$$

with $s=j 2 \pi f$.

The cell is modelled as a rectangular prism discretized in eight elements as shown in the figure (1) :

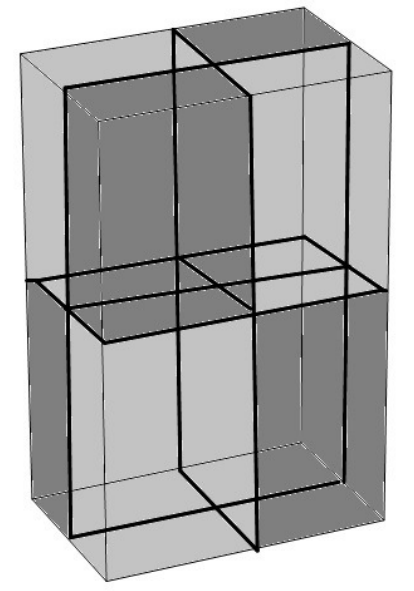

Figure $1:$ Simplified representation of cell discretization

The thermocouple inertia and contact resistance are considered a part of the heat capacity and heat conductivity. The heat transfer coefficient (h) from the cell casing to the ambient is considered constant over the surface of the cell. A different coefficient was calculated for each surface using empirical correlations for free convection (Nusselt and Rayleigh numbers for vertical plates) and thermal conductivity for the top and bottom surfaces (conductivity through busbar and $\log$ connection for the top and through the table for the bottom). An example of energy balance equation for the discrete element $(x, y, z)=(1,1,1)$ is given in equation (6) which is based on equation (5). The dependency on $(s)$ was dropped for simplicity. In this equation, $n$ is the number of element. The conductivity and convection heat transfer coefficients are given by :

$$
\begin{gathered}
k_{x A}=\frac{d y \cdot d z}{d x} k_{x} \\
k_{y A}=\frac{d x \cdot d z}{d y} k_{y} \\
k_{z A}=\frac{d x \cdot d y}{d z} k_{z} \\
h_{A x}=d y \cdot d z \cdot h_{x} \\
h_{A y}=d x \cdot d z \cdot h_{y} \\
h_{A z}=d x \cdot d y \cdot h_{z}
\end{gathered}
$$

Where $d x, d y$ and $d z$ are the dimensions of the element in the $\mathrm{x}, \mathrm{y}$ and $\mathrm{z}$ directions.

Since the inner structure of the cell was unknown, we supposed that the heat generation within the cell was uniform. Then, from equation (6), we can build a system of eight algebraic equations with eight unknowns.

\section{Experimental setup}

A LiFePO $/$ graphite lithium-ion 42 Ah prismatic battery was equipped with type-K thermocouples on different surfaces. Two additional thermocouples were monitoring ambient temperature which was set at approximately $20^{\circ} \mathrm{C}$. The cell was placed in a small insulated chamber to reduce the influence of external temperature variations. 


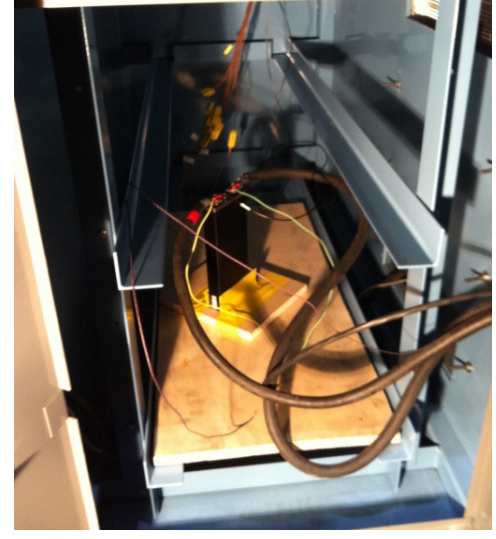

Figure 2 : Cell in test chamber

To raise the cell temperature, a square wave of amplitude 23.9A at a frequency of $16 \mathrm{mHz}$ was applied to the battery using a Bitrode EV/HEV Battery Cell Testing System model MCV. The state of charge (SOC) was set to $50 \%$ before the test. This SOC value was chosen since it is the mean operating value for the target application. The data (voltage, current and temperature) was recorded using an Agilent 34972A data acquisition unit.

\section{Results}

The cell was allowed to reach ambient temperature and OCV condition during the night before the experiment. We applied a square wave with amplitude of 23.9A for 7200 seconds. The figure 3 shows the result of the temperature rise in the centre of the cell on the large face in the time domain averaged on the 125 consecutive samples.

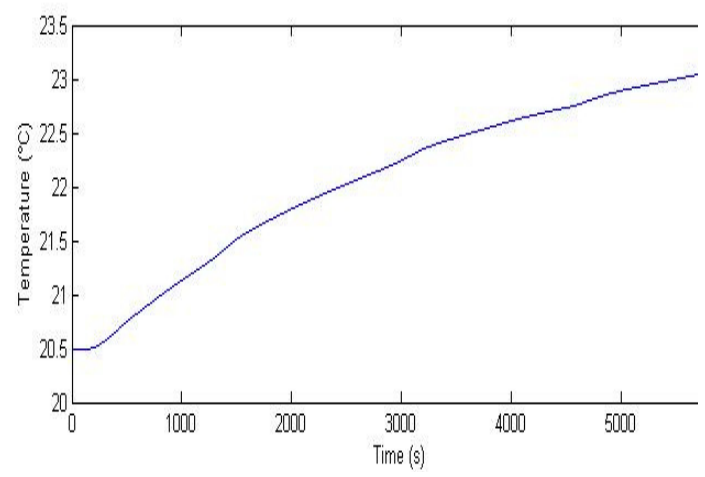

Figure 3 : Temperature rise of the cell with a heat generation of $0.65 \mathrm{~W}$
The heat generation was estimated using equation (x). For a constant current amplitude of $23.9 \mathrm{~A}$, the heat generation within the cell was of $0.65 \mathrm{~W}$.

We applied the Fast Fourier Transform algorithm in Matlab on the heat generation and the temperature signal. By fitting these results in equation (2), we have found the thermal impedance which is presented in figure 4 .

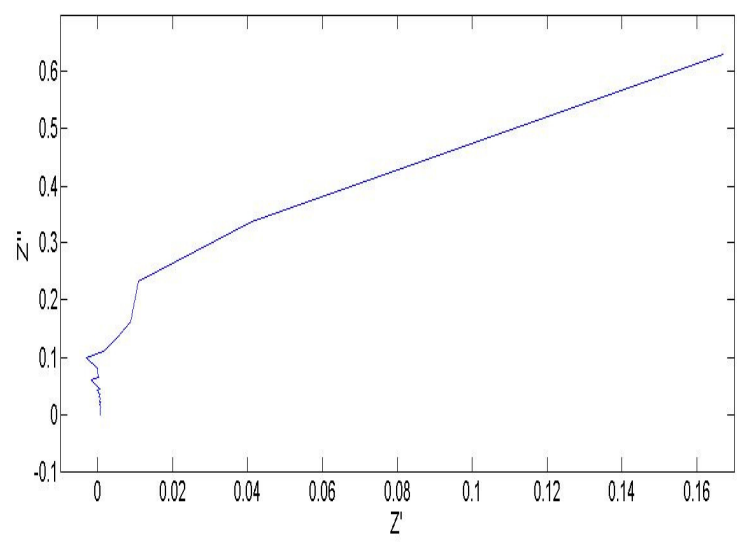

Figure 4 : Nyquist plot of the cell thermal impedance

We tested the cell at four different heat generation frequencies with the frequencydomain method as shown in [2] for validation purposes. The calculated impedance for the two methods were in the same order of magnitude. Unfortunately, the ambient temperature differences between the different tests make it difficult to have a real comparison between the two methods.

To find the thermal parameters of the cell, the measured temperature in the frequency domain was fitted to the temperature obtained from the model with the function fminsearch in Matlab. The optimization function was the complex least square given by equation (13)

$$
\begin{array}{r}
f_{\text {min }}=\sum_{i=1}^{n}\left(T_{\text {meas }}^{\prime}\left(f_{i}\right)-{T^{\prime}}_{\text {model }}\left(f_{i}\right)\right)^{2} \\
+(13) \\
+\left(T^{\prime \prime}{ }_{\text {meas }}\left(f_{i}\right)-T^{\prime \prime}{ }_{\text {model }}\left(f_{i}\right)\right)^{2}
\end{array}
$$


Table 1 gives the fitted parameters results.

Table 1: Fitted thermal parameters

\begin{tabular}{|c|c|c|c|}
\hline $\begin{array}{c}C_{p} \\
(J / k g / K)\end{array}$ & $\begin{array}{c}k_{x} \\
(W / m / K)\end{array}$ & $\begin{array}{c}k_{y} \\
(W / m / K)\end{array}$ & $\begin{array}{c}k_{z} \\
(W / m / K)\end{array}$ \\
\hline 1142 & 2.4 & 0.3 & 0.1 \\
\hline
\end{tabular}

The values in Table 1 are the best fit from all the tests conducted during this experiment. Because of poor control over ambient temperature, even with the insulated chamber, there was no way to reproduce the test correctly.

\section{Discussion}

By comparing the results of table 1 with the literature, we know that the results are in the correct order of magnitude. For example, ref [3] gave a $C_{p}$ of $1020 \mathrm{~J} \mathrm{~kg}^{-1} \mathrm{~K}^{-1}$ and a conductivity perpendicular to the electrode of 0.35 $W \mathrm{~m}^{-1} \mathrm{~K}^{-1}$ for a cylindrical cell which is similar to the result in Table 1 for $C_{p}$ and $k_{y}$. [8] gives four different $C_{p}$, two for cylindrical cells and two for prismatic cells, taken from previous publications ranging between 830 and 1011.

The variation of $C_{p}$ for the different authors is easily explained by the type of cell tested and the test method. For example, the authors of [3] found a difference of $12 \%$ for the thermal conductivity and a difference of $5 \%$ for the heat capacity for the same cell model using two testing methods.

The values of $C_{p}$ and $k_{y}$ are similar to what is found in the literature. There is no available data for the values of $k_{x}$ and $k_{z}$ but we can assume that they are in the correct range as well. The value of $k_{x}$ should be higher since it is parallel to the electrode stack and the value of $k_{z}$ should be smaller since there is a high thermal resistance at the connection of the electrode with the current collector tabs because of the small cross-section.

Some improvements to the test setup should be made to improve the confidence in the test results. First, a better control of the ambient temperature is mandatory. The test reproducibility was compromised because of the ambient temperature variations. A chamber with a greater thermal inertia a better insulation would be needed to make sure there is no influence on the results.

Another improvement would be a better control of the boundary condition. The approximations in free convection have a big influence on the thermal parameters fitting procedure since they are of the same order of magnitude as the thermal conductivity within the cell. We propose to use forced convection which should offer more control on the heat transfer from the casing to the surrounding air. It would also speed up the test process since the cell temperature would reach a steady state value faster.

Improvements to the heat generation modelling would help to increase the precision of the test. The equation ( $\mathrm{x}$ ) used to model the heat generation is valid for ohmic resistance only. We know that this equation is, at best, a correct approximation since the battery cell resistance at the tested frequency is a much more complex system. To reach an heat generation caused only by ohmic resistance, the frequency of the square wave should be much higher. Unfortunately, the testing equipment used (the BitRode cell testing system) is not able to apply current at a square wave frequency higher than $1 \mathrm{~Hz}$, which is still too slow to have an ohmic resistance only. To improve the precision of the test, the heat generation should therefore be calculated by a electro-thermal cell model.

Finally, an increased complexity of the thermal model would increase the precision of the results. The mesh used here with eight elements is coarse and a convergence of the results could probably be detected with increasing complexity of the thermal model.

\section{Conclusion}

This article describes the application of the TIS method in three dimensions to a prismatic cell. In order to find the thermal parameters, a model in three dimensions was described in the frequency domain through the application of the Laplace transform. The data was transferred in the frequency domain through the application of the Fourier transform with the FFT algorithm of Matlab. The frequency domain data was fitted to the model in order to deduce the thermal 
parameters $C_{p}$ and $k_{x}, k_{y}, k_{z}$. The obtained parameters are in the correct order of magnitude but improvements to the test setup should be made to improve the reliability of those values.

We have found that because of the poor ambient temperature control, the results were hardly reproducible. We have also found that, in order to have reliable results with this method for large capacity cells, we need to include a better modelling of the heat generation since it is difficult to apply large current at high frequencies which would allow us to stimulate heat only through ohmic losses.

Subsequent work will include a more precise approximation of the heat generation, a finer model with more elements and improved boundary conditions. The test setup should improve the control over ambient temperature to increase the repeatability of the method.

\section{Acknowledgments}

The author would like to thank MJ EcoPower Hybrid Systems for providing the test setup and the support in the realisation of this project.

\section{References}

[1] Bandhauer et Al., A critical review of thermal issues in Lithium-ion batteries, Journal of the Electrochemical Society, ISSN 1945-7111, 158(2011), R1-R25

[2] J.P. Schmidt et Al., Investigation of the thermal properties of a Li-ion pouch-cell by electrothermal impedance spectroscopy, Journal of Power Sources, ISSN 0378-7753, 196(2011), 8140-8146

[3] M. Fleckenstein et Al., Thermal Impedance Spectroscopy - A method for the thermal characterization of high power battery cells, Journal of Power Sources, ISSN 0378-7753, 223(2013), 259-267

[4] X.Z. Yuan et al, Electrochemical Impedance Spectroscopy in PEM Fuel Cells, ISBN 978-1-84882-845-2, Washington, Island Press, 2005

[5] E. Barsoukov et Al., Thermal impedance spectroscopy for Li-ion batteries using heat-pulse response analysis, Journal of Power Sources, ISSN 0378-7753, 109(2002),313-320
[6] L. Rao and J. Newman, Heat-Generation Rate and General Energy Balance for Insertion Battery Systems, Journal of the Electrochemical Society, ISSN 1945-7111, 144(1997),2697-2704

[7] Incropera et al, Fundamentals of Heat and Mass Transfer, ISBN 978-0-471-45728-2, Danver,MA, John Wiley \& Sons, 2007

[8] M. Shadman Rad et Al., Adaptive thermal modeling of Li-ion batteries, Electrochemica Acta, ISSN 0013-4686, 102(2013),183-195

\section{Author}

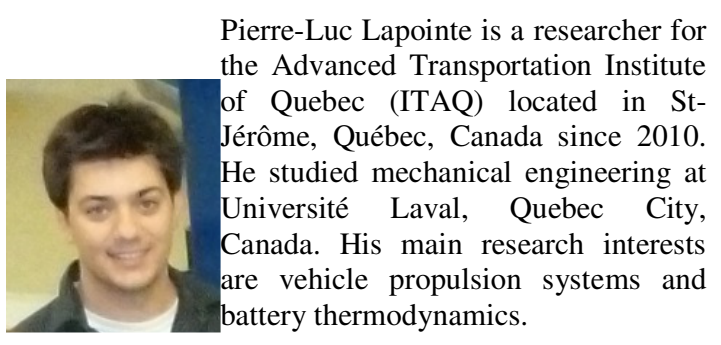

